\title{
The effects of menstrual cycle phase on physical performance in female rugby athletes: A case-study
}

\author{
Francesco S. Sella ${ }^{1}$, Christopher M. Beaven ${ }^{1}$, Stacy T. Sims ${ }^{1,2,3}$, Daniel T. McMaster ${ }^{1}$, Nicholas D. Gill ${ }^{1,4}$, Kim \\ Hébert-Losier ${ }^{1}$
}

${ }^{1}$ Te Huataki Waiora School of Health, University of Waikato Adams Centre for High Performance, Mount Maunganui, New Zealand ${ }^{2}$ Sports Performance Research Institute New Zealand (SPRINZ), AUT University, Auckland, New Zealand

${ }^{3}$ High Performance Sport New Zealand (HPSNZ), Auckland, New Zealand

${ }^{4}$ New Zealand Rugby, Wellington, New Zealand

\section{A R T I C L E I N F O \\ Received: 18.12 .2020 \\ Accepted: 30.08 .2021 \\ Online: 13.10 .2021}

Keywords:

Follicular

Luteal

Testing

Eumenorrhoeic

\begin{abstract}
A B S T R A C T
Limited research exists on the effects of menstrual phase on athletic performance in team sport athletes. In this case-study we investigated the potential effect of menstrual cycle phase on several physical qualities in rugby athletes. Four eumenorrhoeic female rugby athletes completed a battery of physical tests weekly for 5-9 weeks, including 10-m sprint, countermovement (CMJ) and drop (DJ) jumps, isometric mid-thigh pull (IMTP), and Bronco. Concurrently, athletes tracked their menstrual cycle with a smartphone application (FitrWoman ${ }^{T M}$ ). To investigate differences in physical performance between phases, data were allocated into four different menstrual phases at the date of each weekly test. A mixed linear model was created for each physical quality of interest. Mean changes between phases were estimated using magnitude-based inferences with $90 \%$ confidence intervals. Individual differences between the average score for each menstrual phase with the value predicted by the trend of the other three phases were also assessed. At a group-level, possible greater performances were observed in the CMJ during the late luteal phase compared with menstruation, in the DJ during late luteal compared with luteal, and in the IMTP during late luteal compared with follicular to ovulation $(\Delta \%=4.9-7.0 \%)$. A variety of responses were observed between individuals for all the tests conducted. Understanding and accounting for individual responses during the menstrual cycle will likely be beneficial to training prescription and interpreting performance monitoring results.
\end{abstract}

\section{Introduction}

It is well known that the physiology of women is unique and largely influenced by the menstrual cycle (Mujika \& Taipale, 2019). The menstrual cycle is a biological rhythm characterised by the cyclic fluctuations of endogenous sex hormones, such as oestrogen and progesterone. A typical menstrual cycle lasts 2832 days and consists of a follicular phase ( $12-14$ days; low to rising levels of oestrogens and low levels of progesterone), ovulation ( $\sim 1$ day, preceded by an oestrogen surge) and a luteal phase ( $\sim 12-14$ days; high levels of oestrogens and progesterone) (Sims \& Heather, 2018).
While the primary function of oestrogen and progesterone is to support reproduction, the changing concentrations of these hormones across the menstrual cycle have also been found to affect a number of physiological systems, which in turn could have implications on sport performance (Constantini et al., 2005; de Jonge, 2003). However, research findings on this topic are conflicting, with large variance between studies. These equivocal findings are likely attributable to differences in study design, participants' characteristics, number and definition of menstrual cycle phases, phase verification methods, variables measured, and relatively small sample sizes (de Jonge et al., 2019; Julian et al., 2017; McNulty et al., 2020).

\footnotetext{
${ }^{*}$ Corresponding Author: Francesco S. Sella, Te Huataki Waiora School of Health, University of Waikato, New Zealand, fss4@students.waikato.ac.nz
} 
A limited number of studies have investigated the effects of menstrual cycle phase on physical performance in team sport athletes, with most of the research addressing individual sport athletes or untrained people. Previous studies conducted in female soccer athletes revealed unclear and non-significant differences in sprint, repeated sprint ability, and jumping performance between phases (Julian et al., 2017; Somboonwong et al., 2015; Tounsi et al., 2018); however, while Tounsi et al. (2018) observed no differences in the Yo-Yo Intermittent Recovery Test Level 1 between early follicular, late follicular, and luteal phases, Julian et al. (2017) reported possibly better performance in the Yo-Yo Intermittent Recovery Test Level 2 during the early follicular phase compared to mid luteal (effect size, ES $=0.56$ ). The different aerobic and anaerobic contributions to the two tests (Bangsbo et al., 2008) might explain the contrasting results.

Rugby is a high-intensity, intermittent, field-based contact sport that requires players to possess a range of physical qualities, including speed, power, strength, and fitness (Ross et al., 2014). To date, only one study (Miskec et al., 1997) has evaluated the potential influence of menstrual cycle phases in rugby athletes. Specifically, these results highlighted no significant differences between early follicular and luteal phase on anaerobic power output during repeated high-intensity, intermittent exercise on a cycle ergometer. However, no further physical tests were conducted. There is a requirement for more ecologically valid research in this area, and specifically data that are relevant at an individual level. In support of this statement, a recent study addressing elite female rugby athletes has highlighted the importance to develop understanding on the menstrual cycle and considering its impact on training and competition on female rugby athletes (Findlay et al., 2020). Therefore, in this case-study we investigated the potential effect of menstrual cycle phase on several physical qualities in female rugby athletes. Due to the variability observed in the concentration of sex hormones and timing of cycle phases both between- and within-subjects (Haggstrom, 2014; Vescovi, 2011), participants' individual responses to cycle phases were assessed, in addition to average group responses.

\section{Methods}

\subsection{Participants}

Eighteen non-elite female rugby athletes (mean \pm standard deviation: age, $23 \pm 3$ years; height, $1.67 \pm 0.05 \mathrm{~m}$; body mass, 80 $\pm 18 \mathrm{~kg}$ ) from the same New Zealand Provincial Union were recruited to participate. Four athletes fulfilled all inclusion criteria (age, $23 \pm 4$ years; height, $1.69 \pm 0.05 \mathrm{~m}$; body mass, $67 \pm 6 \mathrm{~kg}$; menstrual cycle duration, $30 \pm 4$ days) and were included in the analysis (Figure 1). Inclusion criteria for participation were: being injury free, the absence from any form of contraception for at least six months, having a menstrual cycle duration of 24-35 days before the beginning of the study (Lebrun et al., 1995), and completing at least one testing session in each of the four menstrual cycle phases considered. Written informed consent was obtained from each participant and approval was obtained from the University of Waikato Human Research Ethics Committee (HREC\#2018-10).

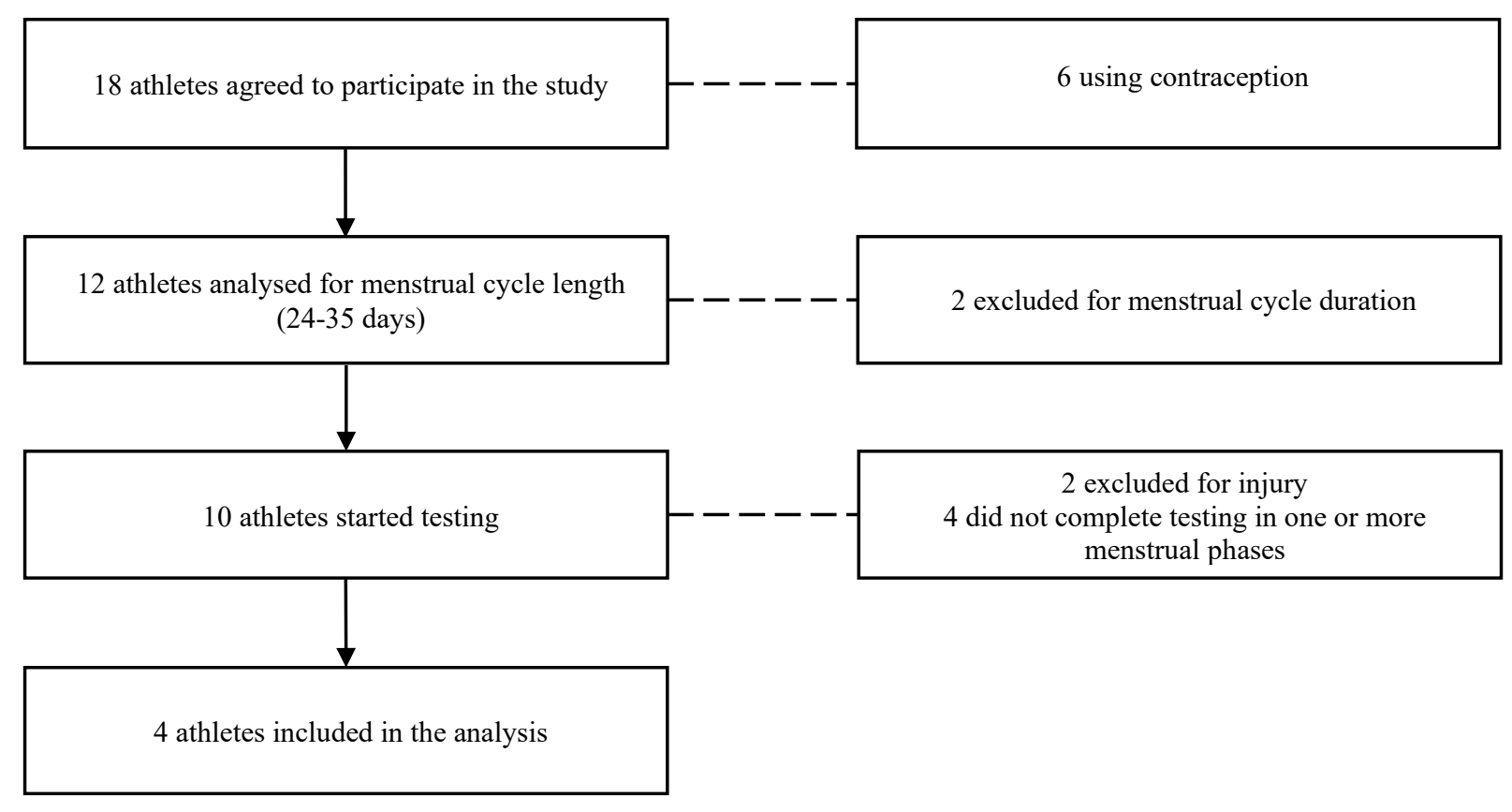

Figure 1: Participant flow chart 


\subsection{Study design}

An observational study design with repeated measures was used. Participants completed a battery of physical tests weekly, for 5-9 consecutive weeks, during the preparation period. Concurrently, they tracked their menstrual cycle daily for the duration of the study.

Physical testing was performed weekly, irrespective of the menstrual phase for a given individual. Participants' data were deidentified to the principal investigators to maintain blinding of the menstrual cycle phase at the time of testing.

A familiarisation session was completed for all testing procedures before the start of the study. The physical assessments were conducted in the same order, on the same day of the week, at the same time of the day (Taylor et al., 2010; Teo et al., 2011) for the duration of the study. Participants were asked to standardise their dietary intake and to abstain from alcohol within the 24 hours before each test.

\subsection{Physical tests}

A 10-minute standardised warm up comprising of dynamic stretches, jogging, running drills, and stride outs was performed before starting the physical testing.

Acceleration. Acceleration abilities were assessed using a 10 $\mathrm{m}$ sprint with split times at 5 and $10 \mathrm{~m}$. The test was completed indoor in running shoes using single beam timing lights (Brower Timing System, Utah, USA). The first gate was set at $0.5 \mathrm{~m}$, while the remaining were set at a height of $0.75 \mathrm{~m}$. Sprint times at each split were measured to the nearest 0.01 second. Participants started each sprint in a standing split position $0.5 \mathrm{~m}$ behind the first gate and were instructed to "run as fast as possible" past the last gate. Each participant was given one warm-up trial followed by three maximal sprints, separated by two min of rest. The repeatability for $10-\mathrm{m}$ sprint times conducted under similar conditions is excellent (intraclass correlation coefficient, ICC = 0.90) (Goodale et al., 2016).

Lower-body power. Body weight countermovement jump (CMJ) and drop jump (DJ) were assessed using an optical measurement system (OptoJump Next, Microgate, Bolzano, Italy) sampling at $1000 \mathrm{~Hz}$. For the CMJ, participants started from an upright standing position with their hands-on hips. Participants were instructed to "bend their knees to a self-selected depth and to jump as high as possible". For the DJ, participants stepped out and dropped off a $30 \mathrm{~cm}$ high box keeping their hands on their hips. Participants were instructed to "minimise time spent in contact with the ground and jump as high as possible as quickly as possible". For both jumps, one warm-up trial was given, followed by three maximal jumps separated by five seconds. CMJ height estimated from flight time and DJ reactive strength ratio (RSR) defined as the ratio between flight time and contact time were considered for analysis. These variables (CMJ height and RSR) have been shown reliable using similar equipment based on coefficient of variation values ( $\mathrm{CV}=2.2$ and $4.2 \%$, respectively) (Byrne et al., 2017; Glatthorn et al., 2011).

Strength. Maximal strength was measured via the isometric mid-thigh pull exercise (IMTP) using a force measurement system comprising of a strain gauge load cell and software package sampling at $1000 \mathrm{~Hz}$ (The Strength Assessment Tool, AUT University, Auckland, NZ). The load cell was anchored to a wooden platform and connected to handles via chains. Testing protocol and position were standardised in agreement with the guidelines of Comfort et al. (e.g., knee angle $125-145^{\circ}$, hip angle 140-150 $)($ Comfort et al., 2019) and kept consistent for each athlete throughout the study. Participants were instructed to pull "as hard and as fast as possible" for three seconds. Verbal encouragement was given throughout the pull. Two sub-maximal IMTP trials at increasing intensities were given, followed by three maximal efforts interspersed by two minutes of passive rest. Peak force $(\mathrm{PF})$ determined as the maximum force generated during the three seconds pull was recorded and considered for the analysis. $\mathrm{PF}$ assessed with a similar device has been found to be reliable (ICC $=0.96$ and CV $=3.1 \%$ ) (James et al., 2017).

Fitness. The $1.2 \mathrm{~km}$ shuttle run test, also known as Bronco test (Kelly \& Wood, 2013) was used as a measure of fitness. The test was performed outdoors, on the same surface, in running shoes. The protocol consists of a continuous 20,40,60 m straight shuttle run, completed five times at maximal intensity (i.e., $20 \mathrm{~m}$ and back, $40 \mathrm{~m}$ and back, $60 \mathrm{~m}$ and back) (Kelly \& Wood, 2013). Total running time was recorded and used for analysis. Excellent testretest reliability of Bronco times has been reported $(\mathrm{ICC}=0.99)$ (Brew \& Kelly, 2014).

\subsection{Menstrual cycle monitoring}

Menstrual cycle information was tracked daily with a smartphone application (FitrWoman ${ }^{\mathrm{TM}}$ ). Participants started the monitoring 10 weeks prior to the start of physical testing to determine their menstrual cycle duration as required by the inclusion criteria. Participants continued to monitor their cycle throughout the 9week duration of the study.

The length of the menstrual cycle was calculated from the first day of menses to the day preceding the next menses. By inputting typical cycle length, period duration, and the date of their last period, FitrWoman ${ }^{\mathrm{TM}}$ estimates menstrual cycle phases. The application divides the menstrual cycle duration in four phases: Phase 1 (menstruation), Phase 2 (follicular to ovulation), Phase 3 (luteal), and Phase 4 (late luteal).

\subsection{Training load and well-being}

The internal training load for all training sessions completed by each participant during the 5 to 9 -week period was calculated using the session-RPE method (Foster et al., 2001). This method quantifies internal training load as the product of the training session rate of perceived exertion (RPE) multiplied by session duration.

The Daily Analysis of Life Demands for Athletes (DALDA) questionnaire (Rushall, 1990) was employed as a measure of wellbeing. The questionnaire contains 34 items to evaluate the sources and symptoms of stress. Each question can be answered either as "worse than normal", "normal", or "better than normal". Participants filled the questionnaire at each testing session before the warm up. 


\subsection{Hydration status}

To minimise the effects of hypohydration on physical performance outcome (McDermott et al., 2017), the hydration status of every participant was verified on each testing day before the warm up. Urine specific gravity (USG) was measured using urine test strips (Combur ${ }^{\circledR-T e s t ~ s t r i p, ~ R o c h e ~ D i a g n o s t i c s) . ~ T h i s ~}$ method has been shown to be valid and reliable ("Combur-Test ${ }^{\circledR}$ strip", 2020; Warren et al., 2018; Zubac et al., 2014). When the USG value was between 1.020 and 1.025 , participants were asked to ingest a $5 \mathrm{ml} / \mathrm{kg}$ of body mass beverage $\left(\mathrm{Na}^{+}: 132 \mathrm{mg} / 100 \mathrm{ml}\right.$, $\mathrm{K}^{+}: 78 \mathrm{mg} / 100 \mathrm{ml}$, CHO: $1.4 \mathrm{~g} / 100 \mathrm{ml}$, $\mathrm{mOsm} / \mathrm{L}: 230 \mathrm{mmol} / \mathrm{L}$ ). With a USG of 1.030 or higher, $10 \mathrm{ml} / \mathrm{kg}$ of body mass of the same beverage was given (Sawka et al., 2007). Furthermore, if the $\mathrm{pH}$ value recorded was 7 or more, 0.005 was added to the USG score (Abbey et al., 2014).

\subsection{Statistical analysis}

At the end of the 9-week period, data were allocated into menstruation, follicular to ovulation, luteal, or late luteal phases at the date of each testing session. Except for the Bronco, the average of the best two scores achieved in the tests at every session was used for analysis.

To investigate differences in physical performance between menstrual phases, physical test scores were log-transformed and analysed using the mixed linear model procedure (Proc Mixed) in the Statistical Analysis System (University Edition of SAS Studio, version 9.4, SAS Institute, Cary NC). A model was created for each physical quality of interest. Menstrual cycle phase, the mean change over the duration of the study, and the weekly training load before each testing session were included in the model as fixed effects. The differences between athletes in the middle of the study and the individual differences in the overall change were entered as random effects with an unstructured covariance matrix to allow these two effects to be correlated.

Mean changes between phases were estimated using magnitude-based inferences with 90\% CI (Hopkins, 2006) and are presented as a percent. Furthermore, the effects of training load on the physical tests results were quantified using the same approach. Quantitative chances of higher or lower differences were evaluated qualitatively as follows: $<0.5 \%$, most unlikely; $0.5-5 \%$, very unlikely; $5-25 \%$, unlikely; $25-75 \%$, possibly; $75-95 \%$, likely; $95-99.5 \%$, very likely; $>99.5 \%$, most likely. If the probabilities of the true value being substantially higher and lower were both $>5 \%$, the difference was termed as unclear.

Individual differences between the mean score for each menstrual phase with the mean predicted by the trend of the other three phases were assessed for each physical quality (Hopkins, 2017). Specifically, the residual obtained from the mixed linear model was used as typical error in percent units. The smallest worthwhile change (SWC $=0.2 \mathrm{x}$ between-subjects $\mathrm{SD}$ ) calculated from previous data collected in our lab was used to establish the smallest important change in percent units. Quantitative chances of higher or lower differences were evaluated qualitatively as follows: $<10 \%$, very unlikely; $10-90 \%$, possible; $>90 \%$, very likely. If the probabilities of the true value being substantially higher and lower were both $>10 \%$, the difference was termed as unclear.

With regards to wellness, DALDA responses were coded as 1 = "worse than normal", 2 = "normal", and 3 = "better than normal" and summed across the 34 items. The total score obtained in the questionnaire by each athlete was then rescaled to range from 0 to 100. Individual differences in the average scores between phases were interpreted as small, moderate, large, very large, and extremely large when reaching thresholds of 10, 30, 50, 70 , and $90 \%$, respectively.

\section{Results}

All data are presented as mean $\pm \mathrm{SD}$, unless otherwise indicated. The maximum variation in cycle length throughout the study was five days. Individual average physical performance results specific to menstrual cycle phase and the number of testing sessions completed in every phase by each participant are reported in Table 1.

Mean differences in physical performance between menstrual cycle phases are reported in Table 2. At a group-level, possible lower CMJ performance was observed in the menstruation phase compared to late luteal $(\Delta \%=-4.9 \%)$. Possible lower DJ performance was observed in the luteal phase compared to late luteal $(\Delta \%=-7.0 \%)$. Furthermore, possible lower IMTP performance was observed in the follicular to ovulation phase compared to late luteal $(\Delta \%=-6.0 \%)$. All other phase differences were possible to very likely trivial or unclear.

Individual differences in physical performance are reported in Table 3. One participant had very likely slower 5-m time in the luteal phase compared with the trend of the other three phases. Another athlete displayed possible greater IMTP performance in the menstruation phase compared with the trend of the other three phases. With regards to the Bronco test, one athlete showed very likely trivial differences between the follicular to ovulation phase with the trend of the other three phases, and between the luteal phase with the trend of the other three phases; furthermore, another athlete displayed a possible trivial difference between the late luteal phase with the trend of the other three phases. The remaining comparisons were unclear or were scored both as a possible substantial increase or decrease and a possible trivial difference.

Training load had a possible to very likely trivial effect on Bronco, CMJ, DJ, 5-m, and 10-m speed scores. An unclear effect was registered for the IMTP. Trivial to small differences were observed in well-being (DALDA) between phases at an individual level (Figure 2).

\section{Discussion}

This is the first study investigating the effects of menstrual cycle phase on several physical qualities in rugby athletes over multiple testing sessions. The inclusion and analysis of participants' individual responses to cycle phase, in addition to the group average responses, also represent a novelty. 
Table 1: Individual average physical performance results specific to menstrual cycle phase across 5 to 9 weeks.

\begin{tabular}{|c|c|c|c|c|c|}
\hline \multirow[t]{2}{*}{ Test } & \multirow[t]{2}{*}{ Phase $^{\mathrm{a}}$} & \multicolumn{4}{|c|}{ Participants } \\
\hline & & 1 & 2 & 3 & 4 \\
\hline \multirow[t]{4}{*}{ 5-m time (s) } & Phase $1(n)$ & $1.14 \pm$ NA $(1)$ & $1.08 \pm$ NA $(1)$ & $1.12 \pm \mathrm{NA}(1)$ & $1.06 \pm 0.00(2)$ \\
\hline & Phase $2(n)$ & $1.15 \pm 0.02$ & $1.08 \pm 0.00$ & $1.12 \pm 0.03$ & $1.07 \pm 0.01$ \\
\hline & Phase $3(n)$ & $1.14 \pm 0.03$ & $1.07 \pm 0.01$ & $1.11 \pm 0.03$ & $1.12 \pm 0.05$ \\
\hline & Phase $4(n)$ & $1.16 \pm \mathrm{NA}(1)$ & $1.07 \pm 0.01$ & $1.12 \pm \mathrm{NA}(1)$ & $1.07 \pm 0.01$ \\
\hline \multirow[t]{4}{*}{ 10-m time (s) } & Phase $1(n)$ & $1.96 \pm$ NA $(1)$ & $1.82 \pm$ NA $(1)$ & $1.95 \pm \mathrm{NA}(1)$ & $1.85 \pm 0.01(2)$ \\
\hline & Phase $2(n)$ & $1.94 \pm 0.01$ & $1.82 \pm 0.02(2)$ & $1.93 \pm 0.04$ & $1.87 \pm 0.01$ \\
\hline & Phase $3(n)$ & $1.94 \pm 0.04$ & $1.83 \pm 0.02(2)$ & $1.92 \pm 0.07$ & $1.89 \pm 0.05$ \\
\hline & Phase $4(n)$ & $1.95 \pm$ NA $(1)$ & $1.81 \pm 0.02(2)$ & $1.93 \pm \mathrm{NA}(1)$ & $1.84 \pm 0.01(2)$ \\
\hline \multirow[t]{4}{*}{ CMJ height $(\mathrm{cm})$} & Phase $1(n)$ & $34.0 \pm$ NA (1) & $35.7 \pm 5.4(2)$ & $29.7 \pm$ NA ( 1$)$ & $35.5 \pm 1.9(2)$ \\
\hline & Phase $2(n)$ & $34.9 \pm 1.7$ & $38.7 \pm \mathrm{NA}(1)$ & $29.2 \pm 2.3(4)$ & $35.2 \pm 2.6(2)$ \\
\hline & Phase $3(n)$ & $33.9 \pm 1.2(3)$ & $38.4 \pm 0.3(2)$ & $30.0 \pm 0.6(3)$ & $33.3 \pm 4.5(2)$ \\
\hline & Phase $4(n)$ & $36.7 \pm \mathrm{NA}(1)$ & $37.8 \pm 0.0(2)$ & $27.9 \pm \mathrm{NA}(1)$ & $36.4 \pm 2.9(2)$ \\
\hline \multirow[t]{4}{*}{ DJ RSR (ratio) } & Phase $1(n)$ & $1.37 \pm \mathrm{NA}(1)$ & $1.65 \pm \mathrm{NA}(1)$ & $1.37 \pm \mathrm{NA}(1)$ & $1.41 \pm 0.07(2)$ \\
\hline & Phase $2(n)$ & $1.32 \pm 0.17$ & $1.55 \pm \mathrm{NA}(1)$ & $1.24 \pm 0.14$ & $1.39 \pm 0.16(2)$ \\
\hline & Phase $3(n)$ & $1.28 \pm 0.11$ & $1.70 \pm 0.09(2)$ & $1.30 \pm 0.08$ & $1.27 \pm \mathrm{NA}(1)$ \\
\hline & Phase $4(n)$ & $1.39 \pm \mathrm{NA}(1)$ & $1.69 \pm 0.06(2)$ & $1.23 \pm \mathrm{NA}(1)$ & $1.50 \pm 0.09$ \\
\hline \multirow[t]{4}{*}{ IMTP PF (N) } & Phase $1(n)$ & $1702 \pm$ NA (1) & $1833 \pm$ NA (1) & $1850 \pm$ NA (1) & $1750 \pm 198(2)$ \\
\hline & Phase $2(n)$ & $1588 \pm 218$ & $1506 \pm 415$ & $1628 \pm 106(3)$ & $2091 \pm 521$ \\
\hline & Phase $3(n)$ & $1630 \pm 113$ & $1538 \pm 136(2)$ & $1599 \pm 123$ & $1949 \pm$ NA $(1)$ \\
\hline & Phase $4(n)$ & $1615 \pm \mathrm{NA}(1)$ & $1748 \pm 74(2)$ & $1745 \pm$ NA $(1)$ & $1772 \pm 592(2)$ \\
\hline \multirow[t]{4}{*}{ Bronco time (s) } & Phase $1(n)$ & $360 \pm$ NA (1) & $310 \pm$ NA (1) & $335 \pm$ NA $(1)$ & $338 \pm 21(2)$ \\
\hline & Phase $2(n)$ & $353 \pm 4(4)$ & $303 \pm$ NA (1) & $346 \pm 13(4)$ & $333 \pm 4(2)$ \\
\hline & Phase $3(n)$ & $355 \pm 5(3)$ & $319 \pm$ NA (1) & $342 \pm 19(3)$ & $338 \pm 1(2)$ \\
\hline & Phase $4(n)$ & $352 \pm \mathrm{NA}(1)$ & $318 \pm 1(2)$ & $339 \pm \mathrm{NA}(1)$ & $331 \pm 6(2)$ \\
\hline
\end{tabular}

Note: ${ }^{\text {a }}$ Phase 1, menstruation; Phase 2, follicular to ovulation; Phase 3, luteal; Phase 4, late luteal. Data are presented as mean \pm SD.

CMJ, countermovement jump; DJ, drop jump; IMTP, isometric mid-thigh pull; $n$, number of testing sessions completed; NA, not applicable; PF, peak force; RSR, reactive strength ratio. 
Sella et al. / The Journal of Sport and Exercise Science, Journal Vol. 5, Issue 5, 310-320 (2021)

Table 2: Mean differences between menstrual cycle phases.

\begin{tabular}{|c|c|c|c|c|c|}
\hline \multirow[t]{2}{*}{ Test } & \multirow[t]{2}{*}{ Phases $^{\mathrm{a}}$} & \multirow[t]{2}{*}{ Difference (\%) } & \multicolumn{2}{|c|}{$90 \%$ confidence limits } & \multirow[t]{2}{*}{ Inference $^{b}$} \\
\hline & & & Lower & Upper & \\
\hline \multirow[t]{6}{*}{ 5-m time (s) } & $\mathrm{Ph} 1-\mathrm{Ph} 2$ & -0.6 & -2.5 & 1.4 & 6/68/26, Unclear \\
\hline & $\mathrm{Ph} 1-\mathrm{Ph} 3$ & -0.5 & -2.5 & 1.4 & 6/68/26, Unclear \\
\hline & $\mathrm{Ph} 1-\mathrm{Ph} 4$ & 0.0 & -2.2 & 2.0 & 14/70/16, Unclear \\
\hline & $\mathrm{Ph} 2-\mathrm{Ph} 3$ & 0.0 & -1.4 & 1.5 & 8/85/7, Unclear \\
\hline & $\mathrm{Ph} 2-\mathrm{Ph} 4$ & 0.5 & -1.3 & 2.3 & 23/72/5, Unclear \\
\hline & $\mathrm{Ph} 3-\mathrm{Ph} 4$ & 0.5 & -1.4 & 2.3 & 23/71/6, Unclear \\
\hline \multirow[t]{6}{*}{ 10-m time (s) } & $\mathrm{Ph} 1-\mathrm{Ph} 2$ & -0.1 & -1.6 & 1.3 & 5/86/9, Unclear \\
\hline & $\mathrm{Ph} 1-\mathrm{Ph} 3$ & 0.2 & -1.4 & 1.7 & 11/83/6, Unclear \\
\hline & $\mathrm{Ph} 1-\mathrm{Ph} 4$ & 0.5 & -1.1 & 2.1 & 19/77/4, Likely $\leftrightarrow$ \\
\hline & $\mathrm{Ph} 2-\mathrm{Ph} 3$ & 0.3 & -0.8 & 1.4 & 7/92/1, Likely $\leftrightarrow$ \\
\hline & $\mathrm{Ph} 2-\mathrm{Ph} 4$ & 0.6 & -0.8 & 2.0 & 21/78/1, Likely $\leftrightarrow$ \\
\hline & $\mathrm{Ph} 3-\mathrm{Ph} 4$ & 0.3 & -1.2 & 1.8 & 14/82/4, Likely $\leftrightarrow$ \\
\hline \multirow[t]{6}{*}{ CMJ height $(\mathrm{cm})$} & $\mathrm{Ph} 1-\mathrm{Ph} 2$ & -2.2 & -6.3 & 2.1 & 1/78/21, Likely $\leftrightarrow$ \\
\hline & $\mathrm{Ph} 1-\mathrm{Ph} 3$ & -2.6 & -6.7 & 1.6 & $0 / 74 / 26$, Possibly $\leftrightarrow \downarrow$ \\
\hline & $\mathrm{Ph} 1-\mathrm{Ph} 4$ & -4.9 & -9.2 & -0.4 & 0/39/61, Possibly $\downarrow \leftrightarrow$ \\
\hline & $\mathrm{Ph} 2-\mathrm{Ph} 3$ & -0.4 & -3.8 & 3.1 & 2/94/4, Likely $\leftrightarrow$ \\
\hline & $\mathrm{Ph} 2-\mathrm{Ph} 4$ & -2.7 & -6.8 & 1.5 & 0/72/28, Possibly $\leftrightarrow \downarrow$ \\
\hline & $\mathrm{Ph} 3-\mathrm{Ph} 4$ & -2.3 & -6.5 & 2.0 & 1/76/23, Likely $\leftrightarrow$ \\
\hline \multirow[t]{6}{*}{ DJ RSR (ratio) } & $\mathrm{Ph} 1-\mathrm{Ph} 2$ & 1.5 & -6.2 & 9.6 & 15/79/6, Unclear \\
\hline & $\mathrm{Ph} 1-\mathrm{Ph} 3$ & 3.0 & -4.4 & 11.0 & 24/74/2, Possibly $\leftrightarrow$ \\
\hline & $\mathrm{Ph} 1-\mathrm{Ph} 4$ & -4.2 & -11.6 & 3.7 & $2 / 63 / 35$, Possibly $\leftrightarrow \downarrow$ \\
\hline & $\mathrm{Ph} 2-\mathrm{Ph} 3$ & 1.5 & -4.7 & 8.1 & 11/86/3, Likely $\leftrightarrow$ \\
\hline & $\mathrm{Ph} 2-\mathrm{Ph} 4$ & -5.6 & -12.1 & 1.4 & $1 / 53 / 46$, Possibly $\leftrightarrow \downarrow$ \\
\hline & $\mathrm{Ph} 3-\mathrm{Ph} 4$ & -7.0 & -13.2 & -0.3 & 0/39/61, Possibly $\downarrow \leftrightarrow$ \\
\hline \multirow[t]{6}{*}{ IMTP PF (N) } & $\mathrm{Ph} 1-\mathrm{Ph} 2$ & 5.1 & -4.3 & 15.5 & 59/35/6, Unclear \\
\hline & $\mathrm{Ph} 1-\mathrm{Ph} 3$ & 3.0 & -6.2 & 13.0 & 44/45/11, Unclear \\
\hline & $\mathrm{Ph} 1-\mathrm{Ph} 4$ & -1.1 & -10.5 & 9.2 & 20/47/33, Unclear \\
\hline & $\mathrm{Ph} 2-\mathrm{Ph} 3$ & -2.1 & -9.2 & 5.4 & 10/55/35, Unclear \\
\hline & $\mathrm{Ph} 2-\mathrm{Ph} 4$ & -6.0 & -13.6 & 2.4 & $3 / 29 / 68$, Possibly $\downarrow \leftrightarrow$ \\
\hline & $\mathrm{Ph} 3-\mathrm{Ph} 4$ & -4.0 & -11.9 & 4.7 & 7/41/52, Unclear \\
\hline \multirow[t]{6}{*}{ Bronco time (s) } & $\mathrm{Ph} 1-\mathrm{Ph} 2$ & 0.4 & -7.8 & 9.3 & 14/74/12, Unclear \\
\hline & $\mathrm{Ph} 1-\mathrm{Ph} 3$ & 0.2 & -2.3 & 2.7 & 3/95/2, Very likely $\leftrightarrow$ \\
\hline & $\mathrm{Ph} 1-\mathrm{Ph} 4$ & -0.4 & -3.8 & 3.1 & 5/87/8, Likely $\leftrightarrow$ \\
\hline & $\mathrm{Ph} 2-\mathrm{Ph} 3$ & -0.2 & -5.2 & 5.0 & 8/84/8, Unclear \\
\hline & $\mathrm{Ph} 2-\mathrm{Ph} 4$ & -0.8 & -3.1 & 1.5 & 1/94/5, Likely $\leftrightarrow$ \\
\hline & $\mathrm{Ph} 3-\mathrm{Ph} 4$ & -0.6 & -3.1 & 1.9 & 1/94/5, Likely $\leftrightarrow$ \\
\hline
\end{tabular}

Note: ${ }^{a}$ Phase $1(\mathrm{Ph} 1)$, menstruation; Phase $2(\mathrm{Ph} 2)$, follicular to ovulation; Phase $3(\mathrm{Ph} 3)$, luteal; Phase $4(\mathrm{Ph} 4)$, late luteal. ${ }^{\mathrm{b}}$ Likelihood (\%): increase/trivial/decrease. $\leftrightarrow$ Trivial; $\downarrow$ Substantial decrease. CMJ, countermovement jump; DJ, drop jump: IMTP, isometric mid-thigh pull; PF, peak force; RSR, reactive strength ratio. 
Table 3: Individual menstrual cycle phase differences.

\begin{tabular}{|c|c|c|c|c|c|}
\hline \multirow[t]{3}{*}{ Test } & \multirow[t]{3}{*}{ Phases $^{\mathrm{a}}$} & \multicolumn{3}{|c|}{ Participants } & \multirow[b]{2}{*}{4} \\
\hline & & 1 & 2 & 3 & \\
\hline & & Inference $^{\mathrm{b}}$ & Inference $^{b}$ & Inference $^{b}$ & Inference $^{\mathrm{b}}$ \\
\hline \multirow[t]{4}{*}{ 5-m time (s) } & Ph 1 - Trend & 20/38/42, Unclear & 38/45/17, Unclear & 41/42/17, Unclear & 7/39/54, Possible $\downarrow \leftrightarrow$ \\
\hline & $\mathrm{Ph} 2$ - Trend & 12/49/39, Unclear & 33/54/14, Unclear & 31/51/17, Unclear & 39/41/20, Unclear \\
\hline & Ph 3 - Trend & 13/61/26, Unclear & 12/60/29, Unclear & 13/56/31, Unclear & 96/3/0, Very likely $\uparrow$ \\
\hline & Ph 4 - Trend & 41/43/16, Unclear & 12/61/27, Unclear & 22/35/43, Unclear & $7 / 35 / 58$, Possible $\downarrow \leftrightarrow$ \\
\hline \multirow[t]{4}{*}{ 10-m time (s) } & Ph 1 - Trend & 39/47/14, Unclear & 32/54/15, Unclear & $52 / 40 / 8$, Possible $\uparrow \leftrightarrow$ & 13/61/26, Unclear \\
\hline & $\mathrm{Ph} 2$ - Trend & 17/59/24, Unclear & 21/65/14, Unclear & 20/51/29, Unclear & 51/44/5, Possible $\uparrow \leftrightarrow$ \\
\hline & Ph 3 - Trend & 2/71/27, Possible $\leftrightarrow \downarrow$ & 19/70/11, Unclear & $17 / 62 / 21$, Unclear & 69/30/1, Possible $\uparrow \leftrightarrow$ \\
\hline & $\mathrm{Ph} 4$ - Trend & 26/55/19, Unclear & 6/67/27, Possible $\leftrightarrow \downarrow$ & 26/38/37, Unclear & $1 / 32 / 67$, Possible $\downarrow \leftrightarrow$ \\
\hline \multirow[t]{4}{*}{ CMJ height $(\mathrm{cm})$} & Ph 1 - Trend & 5/37/59, Possible $\downarrow \leftrightarrow$ & 0/28/72, Possible $\downarrow \leftrightarrow$ & 21/60/19, Unclear & 15/67/18, Unclear \\
\hline & Ph 2 - Trend & 23/63/14, Unclear & $6 / 36 / 58$, Possible $\downarrow \leftrightarrow$ & $2 / 83 / 15$, Possible $\leftrightarrow \downarrow$ & $1 / 36 / 62$, Possible $\downarrow \leftrightarrow$ \\
\hline & Ph 3 - Trend & $2 / 52 / 45$, Possible $\leftrightarrow \downarrow$ & 72/28/1, Possible $\uparrow \leftrightarrow$ & 29/62/9, Possible $\leftrightarrow \uparrow$ & $5 / 56 / 39$, Possible $\leftrightarrow \downarrow$ \\
\hline & $\mathrm{Ph} 4$ - Trend & $73 / 26 / 1$, Possible $\uparrow \leftrightarrow$ & 21/66/13, Unclear & 19/49/32, Unclear & $76 / 24 / 0$, Possible $\uparrow \leftrightarrow$ \\
\hline \multirow[t]{4}{*}{ DJ RSR (ratio) } & Ph 1 - Trend & 11/41/48, Unclear & 32/55/13, Unclear & 50/43/7, Possible $\uparrow \leftrightarrow$ & 23/55/22, Unclear \\
\hline & $\mathrm{Ph} 2$ - Trend & 31/61/8, Possible $\leftrightarrow \uparrow$ & 7/44/49, Possible $\downarrow \leftrightarrow$ & 3/70/27, Possible $\leftrightarrow \downarrow$ & 12/52/36, Unclear \\
\hline & Ph 3 - Trend & 3/44/53, Possible $\downarrow \leftrightarrow$ & 20/62/18, Unclear & 34/42/23, Unclear & $2 / 23 / 75$, Possible $\downarrow \leftrightarrow$ \\
\hline & Ph 4 - Trend & $72 / 26 / 2$, Possible $\uparrow \leftrightarrow$ & $17 / 74 / 9$, Possible $\leftrightarrow \uparrow$ & 26/44/29, Unclear & $85 / 15 / 0$, Possible $\uparrow \leftrightarrow$ \\
\hline \multirow[t]{4}{*}{ IMTP PF (N) } & Ph 1 - Trend & 44/24/32, Unclear & 44/26/30, Unclear & $89 / 8 / 3$, Possible $\uparrow$ & 4/18/79, Possible $\downarrow \leftrightarrow$ \\
\hline & $\mathrm{Ph} 2$ - Trend & 14/37/50, Unclear & 10/17/73, Unclear & 13/35/52, Unclear & 56/26/19, Unclear \\
\hline & Ph 3 - Trend & 27/37/36, Unclear & $32 / 31 / 37$, Unclear & $10 / 21 / 68$, Unclear & 51/26/22, Unclear \\
\hline & Ph 4 - Trend & 42/28/31, Unclear & $81 / 16 / 3$, Possible $\uparrow \leftrightarrow$ & 47/24/29, Unclear & 44/27/29, Unclear \\
\hline \multirow[t]{4}{*}{ Bronco time (s) } & Ph 1 - Trend & 27/66/7, Possible $\leftrightarrow \uparrow$ & 15/71/14, Unclear & 4/53/43, Possible $\leftrightarrow \downarrow$ & 17/79/3, Possible $\leftrightarrow \uparrow$ \\
\hline & Ph 2 - Trend & 5/94/2, Very likely $\leftrightarrow$ & 5/53/42, Possible $\leftrightarrow \downarrow$ & $27 / 66 / 7$, Possible $\leftrightarrow \uparrow$ & $3 / 60 / 37$, Possible $\leftrightarrow \downarrow$ \\
\hline & Ph 3 - Trend & 5/90/4, Very likely $\leftrightarrow$ & 21/70/9, Possible $\leftrightarrow \uparrow$ & 13/75/12, Unclear & $42 / 56 / 3$, Possible $\leftrightarrow \uparrow$ \\
\hline & $\mathrm{Ph} 4$ - Trend & 10/72/18, Unclear & 5/85/10, Possible $\leftrightarrow$ & 18/53/30, Unclear & 5/72/23, Possible $\leftrightarrow \downarrow$ \\
\hline
\end{tabular}

Note: ${ }^{\text {a Phase }} 1$ (Ph1), menstruation; Phase 2 (Ph2), follicular to ovulation; Phase 3 (Ph3), luteal; Phase 4 (Ph4), late luteal. ${ }^{b}$ Likelihood (\%): increase/trivial/decrease. $\uparrow$ Substantial increase; $\leftrightarrow$ Trivial; $\downarrow$ Substantial decrease. CMJ, countermovement jump; DJ, drop jump; IMTP, isometric mid-thigh pull; PF, peak force; RSR, reactive strength ratio; Trend, value predicted by the trend of the other three phases. 


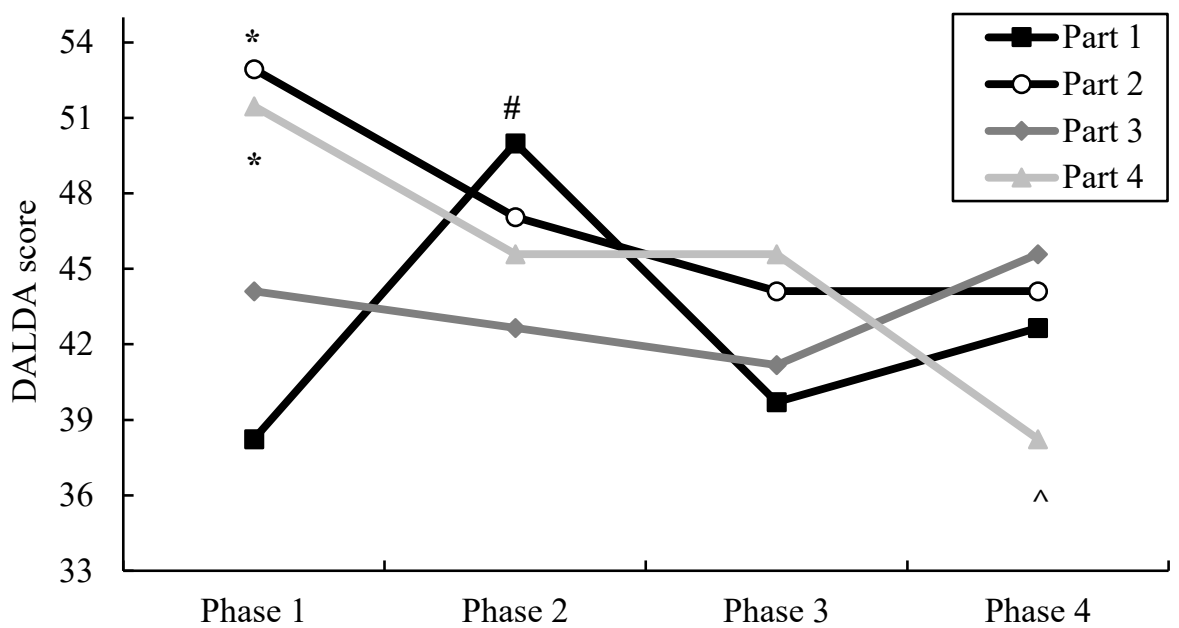

Figure 2: DALDA score specific to each participant. Data are presented as rescaled mean scores for each menstrual cycle phase. Phase 1, menstruation; Phase 2, follicular to ovulation; Phase 3, luteal; Phase 4, late luteal. * = Small difference with Phase 2, Phase 3, and Phase 4. $\#=$ Small difference with Phase 1 , Phase 3 , and Phase $4 .^{\wedge}=$ Small difference with Phase 2 and Phase 3.

In female rugby athletes, possibly greater CMJ performance was observed in the late luteal phase compared with the menstruation phase, with possible to likely trivial changes observed between the other phases. Previous research conducted on female soccer athletes reported unclear differences in CMJ height between early follicular and mid luteal phase (Julian et al., 2017). The different study design, menstrual cycle phases considered, and phase verification methods might explain the conflicting results. In the current study, possibly greater DJ performance was observed in the late luteal phase compared with the luteal phase, with the remaining phase comparisons showing possible to likely trivial or unclear differences. No other studies have investigated the effects of menstrual cycle performance on DJ in team sport athletes. However, previous research reported no significant differences in the five-jump test (Tounsi et al., 2018) between the early follicular, late follicular, and luteal phase in female soccer athletes.

Possible greater peak force was observed in the IMTP in the late luteal phase compared with the follicular to ovulation phase in rugby athletes. Unclear differences were observed in other phase comparisons. To the authors' knowledge, no other study has evaluated the influence of menstrual cycle on maximal isometric strength in team sport athletes using multi-joint exercises (i.e., IMTP). A study (dos Santos Andrade et al., 2017) performed on female soccer athletes found the hamstring/quadriceps peak torque strength balance ratio for the non-dominant limb was significantly lower $(p=0.01)$ during follicular phase compared to the luteal phase, which might have implications in terms of lowerlimb and anterior cruciate ligament injury risk. In contrast, previous research (Hertel et al., 2006) assessing isokinetic concentric strength in female college students (i.e., soccer and cheerleading) found no substantial fluctuations in hamstring and quadriceps muscle strength across the menstrual cycle at grouplevel.
Unclear differences were observed across the menstrual cycle in 5-m sprint time in rugby athletes. Furthermore, unclear differences and likely trivial differences were observed between phases in 10-m sprint time. While the trivial differences highlight the absence of a substantial change in performance between phases, the unclear differences are likely attributed to the small sample size employed in this study (Buchheit, 2018). When considering the existing research on team sports, previous studies addressing female soccer athletes showed unclear and nonsignificant differences between early follicular and mid luteal phases in 5, 10, $30 \mathrm{~m}$ sprint times (Julian et al., 2017), and in 40yard $(\sim 37 \mathrm{~m})$ sprint time (Somboonwong et al., 2015). Furthermore, in female soccer athletes, no significant differences were observed between early follicular, late follicular, and luteal phase in a repeated shuttle-sprint ability test (Tounsi et al., 2018).

Likely to very likely trivial or unclear menstrual phase differences characterised the Bronco test. No significant differences in fitness performance were reported between phases in female rugby (Miskec et al., 1997) and soccer athletes (Julian et al., 2017; Tounsi et al., 2018) in previous research. However, in the study of Julian et al. (2017), female soccer athletes covered possibly greater distance in the Yo-Yo Intermittent Recovery Test Level 2 during the early follicular phase compared to mid luteal $(\mathrm{ES}=0.56)$. These findings contrast the results of this study, and may be explained by the different menstrual phases considered and the different tests employed (Sella et al., in press).

To date, most of the research in this area has focused on the average effects of menstrual cycle on physical performance at group-level, without considering and analysing the individual responses. However, it is known that a large inter- and intraindividual variability exist in the concentration of sex hormones and timing of cycle event (Haggstrom, 2014; Vescovi, 2011) that could potentially affect women differently. Therefore, tracking 
individual performance changes across the different phases of the cycle proves to be critical for athletes and coaches. For example, in this study, no clear differences were observed in 5-m sprint time across the menstrual cycle at group-level. However, one athlete showed very likely slower 5 -m time in the luteal phase compared with the trend of the other three phases. These observations suggest impairment in her short acceleration abilities in the luteal phase, which in turn could affect game performance (Clarke et al., 2017). Another athlete displayed possibly greater peak force in the IMTP exercise in the menstruation phase compared with the trend of the other phases, despite no clear differences were observed at group-level between the menstruation phase with the other phases; therefore, further highlighting the need to account for individual performance differences across the menstrual cycle. In collision-based sports such as rugby, high levels of muscular strength are thought to be important to success (Ross et al., 2014); in particular, peak force assessed in the IMTP appears to be associated with performance in numerous athletic tasks in a variety of athletes (Comfort et al., 2019).

It is worth noting that several phase comparisons at grouplevel and at individual-level, resulted both as a possible substantial increase or decrease and a possible trivial difference. These findings are explained by the magnitude of the SWCs and typical errors (TEs) of the physical tests conducted (TE $\sim$ SWC or TE $>$ SWC), and indicate that the ability to make firm conclusions is limited unless the probability of a substantial change is high. Throughout the study, the weekly training load completed before each testing session did not substantially affect test scores. Trivial to small differences were observed between phases in well-being on the day of testing.

It is important to highlight some limitations of the present study. Firstly, because of the long duration of the investigation, we did not measure oestrogens and progesterone concentrations to verify menstrual cycle phase and no ovulation testing was conducted. Instead, we opted for a designated smartphone application. Compared to direct hormones measurements, this method is a practical, time-efficient and cost-effective alternative to monitor the menstrual cycle (i.e., duration and phase prediction) in a team environment. However, it does not allow distinguishing between ovulatory and anovulatory or luteal phase-deficient cycles, nor does it allow for monitoring the daily variation of hormones between individuals (de Jonge et al., 2019). In addition, knowledge of hormones concentration specific to menstrual cycle phase could have also assisted in explaining performance differences at individual-level between- and within-menstrual cycle phases. Therefore, additional research is required to investigate the validity of this alternative method to monitor menstrual cycle (Julian \& Sargent, 2020). While not addressed in the current study, recovery post-exercise may also differ between phases and impact on overall training outcomes.

Out of the participants that were screened for eligibility $(n=$ 18 ), only 4 completed the study ( $22 \%$, Figure 1$)$. Large degrees of drop-out rates (75 and $82 \%$ ) were also observed in previous research conducted in soccer athletes (Julian et al., 2017; Julian et al., 2020), highlighting one of the challenges in conducting this type of research with athletic population in applied settings.

To improve the quality of menstrual cycle research, further studies are required to address the above limitations. In addition, similar to the study of Julian et al. (2020) in soccer, future research is needed to assess the influence of menstrual cycle directly on rugby and other sports' match activities and determinants of success. Lastly, given the high prevalence of contraception in female athletes (Rechichi et al., 2009), the effects of different hormonal profiles should be also considered.

\section{Conclusions}

In female rugby athletes, possibly greater performances were observed in the CMJ, DJ, and IMTP in the late luteal phase compared with the menstruation, luteal, and follicular to ovulation phases. However, a large variety of responses were observed at an individual-level.

Including menstrual cycle monitoring and understanding the potential effects of cycle phase on physical performance in rugby and other team sports could be of interest for coaches and practitioners. In particular, assessing and accounting for athletes' individual changes during menstrual cycle phases will likely be beneficial for interpreting monitoring results and training prescription.

\section{Conflict of Interest}

The authors declare no conflict of interests.

\section{Acknowledgment}

The authors would like to thank all the athletes taking part in the study. Furthermore, the authors would like to thank Danelle Stevens, Rodney Gibbs, Conor McNeill, Ivana Hanzlíková for their help during data collection, and Prof. Will Hopkins for his statistical support in the preparation of this manuscript.

\section{References}

Abbey, B. M., Heelan, K. A., Brown, G. A., \& Bartee, R. T. (2014). Validity of HydraTrend reagent strips for the assessment of hydration status. The Journal of Strength \& Conditioning Research, 28(9), 2634-2639. https://doi.org/10.1519/JSC.0000000000000430

Bangsbo, J., Iaia, F. M., \& Krustrup, P. (2008). The Yo-Yo intermittent recovery test: A useful tool for evaluation of physical performance in intermittent sports. Sports Medicine, 38(1), 37-51. https://doi.org/10.2165/00007256-20083801000004

Brew, D. J., \& Kelly, V. G. (2014). The reliability of the $1.2 \mathrm{~km}$ shuttle run test for intermittent sport athletes. Journal of Australian Strength and Conditioning, 22(5), 127-131.

Buchheit, M. (2018). Trivial effects are clearly important. Sport Performance \& Science Reports, 1, 1-4

Byrne, D. J., Browne, D. T., Byrne, P. J., \& Richardson, N. (2017). Interday reliability of the reactive strength index and optimal drop height. The Journal of Strength \& Conditioning Research, 31(3), 721-726. https://doi.org/10.1519/JSC.0000000000001534

Clarke, A. C., Anson, J. M., \& Pyne, D. B. (2017). Game movement demands and physical profiles of junior, senior and 


\section{Sella et al. / The Journal of Sport and Exercise Science, Journal Vol. 5, Issue 5, 310-320 (2021)}

elite male and female rugby sevens players. Journal of Sports Sciences, 35(8), 727-733.

https://doi.org/10.1080/02640414.2016.1186281

Combur-Test ${ }^{\circledR} \quad$ strip. $\quad$ Available

at:

https://diagnostics.roche.com/global/en/products/instruments/ combur_chemstripnephurnitur.html. Accessed: October 15, 2020

Comfort, P., Dos' Santos, T., Beckham, G. K., Stone, M. H., Guppy, S. N., \& Haff, G. G. (2019). Standardization and methodological considerations for the isometric midthigh pull. Strength \& Conditioning Journal, 41(2), 57-79. https://doi.org/10.1519/SSC.0000000000000433

Constantini, N. W., Dubnov, G., \& Lebrun, C. M. (2005). The menstrual cycle and sport performance. Clinics in Sports Medicine, 24(2), e51-82.

https://doi.org/10.1016/j.csm.2005.01.003

de Jonge, X. A. K. J. (2003). Effects of the menstrual cycle on exercise performance. Sports Medicine, 33(11), 833-851. https://doi.org/10.2165/00007256-200333110-00004

de Jonge, X. A. K. J., Thompson, B., \& Han, A. (2019). Methodological recommendations for menstrual cycle research in sports and exercise. Medicine \& Science in Sports \& Exercise, 51(12), 2610-2617.

https://doi.org/10.1249/MSS.0000000000002073

dos Santos Andrade, M., Mascarin, N. C., Foster, R., de Jármy di Bella, Z. I., Vancini, R. L., \& de Lira, C. A. B. (2017). Is muscular strength balance influenced by menstrual cycle in female soccer players? The Journal of Sports Medicine and Physical Fitness, 57(6), 859-864.

https://doi.org/10.23736/S0022-4707.16.06290-3

Findlay, R. J., Macrae, E. H. R., Whyte, I. Y., Easton, C., \& Forrest Née Whyte, L. J. (2020). How the menstrual cycle and menstruation affect sporting performance: experiences and perceptions of elite female rugby players. British Journal of Sports Medicine, 54(18), 1108-1113.

https://doi.org/10.1136/bjsports-2019-101486

Foster, C., Florhaug, J. A., Franklin, J., Gottschall, L., Hrovatin, L. A., Parker, S., . . Dodge, C. (2001). A new approach to monitoring exercise training. The Journal of Strength \& Conditioning Research, 15(1), 109-115.

Glatthorn, J. F., Gouge, S., Nussbaumer, S., Stauffacher, S., Impellizzeri, F. M., \& Maffiuletti, N. A. (2011). Validity and reliability of Optojump photoelectric cells for estimating vertical jump height. The Journal of Strength \& Conditioning Research, 25(2), 556-560.

https://doi.org/10.1519/JSC.0b013e3181ccb18d

Goodale, T. L., Gabbett, T., Stellingwerff, T., Tsai, M. C., \& Sheppard, J. M. (2016). Relationship between physical qualities and minutes played in international women's rugby sevens. International Journal of Sports Physiology and Performance, 11(4), 489-494.

https://doi.org/10.1123/ijspp.2014-0509

Haggstrom, M. (2014). Reference ranges for estradiol, progesterone, luteinizing hormone and follicle-stimulating hormone during the menstrual cycle. WikiJournal of Medicine, 1(1), 1-5. https://doi.org/10.15347/wjm/2014.001

Hertel, J., Williams, N. I., Olmsted-Kramer, L. C., Leidy, H. J., \& Putukian, M. (2006). Neuromuscular performance and knee laxity do not change across the menstrual cycle in female athletes. Knee Surgery, Sports Traumatology, Arthroscopy, 14(9), 817-822. https://doi.org/10.1007/s00167-006-0047-4

Hopkins, W. G. (2006). A spreadsheet for combining outcomes from several subject groups. Sportscience, 10, 50-53.

Hopkins, W. G. (2017). A spreadsheet for monitoring an individual's changes and trend. Sportscience, 21, 5-9.

James, L. P., Roberts, L. A., Haff, G. G., Kelly, V. G., \& Beckman, E. M. (2017). Validity and reliability of a portable isometric mid-thigh clean pull. The Journal of Strength \& Conditioning Research, 31(5), 1378-1386.

https://doi.org/10.1519/JSC.0000000000001201

Julian, R., Hecksteden, A., Fullagar, H. H., \& Meyer, T. (2017). The effects of menstrual cycle phase on physical performance in female soccer players. PloS One, 12(3). https://doi.org/10.1371/journal.pone.0173951

Julian, R., \& Sargent, D. (2020). Periodisation: tailoring training based on the menstrual cycle may work in theory but can they be used in practice? Science and Medicine in Football, 4(4), 253-254. https://doi.org/10.1080/24733938.2020.1828615

Julian, R., Skorski, S., Hecksteden, A., Pfeifer, C., Bradley, P. S., Schulze, E., \& Meyer, T. (2020). Menstrual cycle phase and elite female soccer match-play: Influence on various physical performance outputs. Science and Medicine in Football, 5(2), 97-104. https://doi.org/10.1080/24733938.2020.1802057

Kelly, V., \& Wood, A. (2013). The correlation between the 30-15 intermittent fitness test and a novel test of running performance. Journal of Australian Strength Conditioning, 21(S1), 91-94.

Lebrun, C. M., McKenzie, D. C., Prior, J. C., \& Taunton, J. E. (1995). Effects of menstrual cycle phase on athletic performance. Medicine \& Science in Sports \& Exercise, 27(3), 437-444.

McDermott, B. P., Anderson, S. A., Armstrong, L. E., Casa, D. J., Cheuvront, S. N., Cooper, L., . . . Roberts, W. O. (2017). National Athletic Trainers' Association position statement: Fluid replacement for the physically active. Journal of Athletic Training, 52(9), 877-895. https://doi.org/10.4085/1062-605052.9.02

McNulty, K. L., Elliott-Sale, K. J., Dolan, E., Swinton, P. A., Ansdell, P., Goodall, S., . . Hicks, K. M. (2020). The effects of menstrual cycle phase on exercise performance in eumenorrheic women: A systematic review and meta-analysis. Sports Medicine, 50(10), 1813-1827. https://doi.org/10.1007/s40279-020-01319-3

Miskec, C. M., Potteiger, J. A., Nau, K. L., \& Zebas, C. J. (1997). Do varying environmental and menstrual cycle conditions affect anaerobic power output in female athletes? The Journal of Strength \& Conditioning Research, 11(4), 219-223.

Mujika, I., \& Taipale, R. S. (2019). Sport science on women, women in sport science. International Journal of Sports Physiology and Performance, 14(8), 1013-1014. https://doi.org/10.1123/ijspp.2019-0514

Rechichi, C., Dawson, B., \& Goodman, C. (2009). Athletic performance and the oral contraceptive. International Journal of Sports Physiology and Performance, 4(2), 151-162. https://doi.org/10.1123/ijspp.4.2.151

Ross, A., Gill, N., \& Cronin, J. (2014). Match analysis and player characteristics in rugby sevens. Sports Medicine, 44(3), 357367. https://doi.org/10.1007/s40279-013-0123-0 
Sella et al. / The Journal of Sport and Exercise Science, Journal Vol. 5, Issue 5, 310-320 (2021)

Rushall, B. S. (1990). A tool for measuring stress tolerance in elite athletes. Journal of Applied Sport Psychology, 2(1), 51-66. https://doi.org/10.1080/10413209008406420

Sawka, M. N., Burke, L. M., Eichner, E. R., Maughan, R. J., Montain, S. J., \& Stachenfeld, N. S. (2007). American College of Sports Medicine position stand. Exercise and fluid replacement. Medicine \& Science in Sports \& Exercise, 39(2), 377-390. https://doi.org/10.1249/mss.0b013e31802ca597

Sella, F. S., Hébert-Losier, K., Beaven, C. M., McMaster, D. T., Harvey, M., \& Gill, N. D. (in press). Performance in the 1.2 $\mathrm{km}$ shuttle run test reflects fitness capacities in rugby players. Journal of Australian Strength and Conditioning.

Sims, S. T., \& Heather, A. K. (2018). Myths and methodologies: Reducing scientific design ambiguity in studies comparing sexes and/or menstrual cycle phases. Experimental physiology, 103(10), 1309-1317. https://doi.org/10.1113/EP086797

Somboonwong, J., Chutimakul, L., \& Sanguanrungsirikul, S. (2015). Core temperature changes and sprint performance of elite female soccer players after a 15 -minute warm-up in a hothumid environment. The Journal of Strength \& Conditioning Research, 29(1), 262-269. https://doi.org/10.1097/JSC.0000000000000235

Taylor, K. L., Cronin, J., Gill, N. D., Chapman, D. W., \& Sheppard, J. (2010). Sources of variability in iso-inertial jump assessments. International Journal of Sports Physiology and Performance, 5(4), 546-558. https://doi.org/10.1123/ijspp.5.4.546

Teo, W., Newton, M. J., \& McGuigan, M. R. (2011). Circadian rhythms in exercise performance: Implications for hormonal and muscular adaptation. Journal of Sports Science \& Medicine, 10(4), 600-606.

Tounsi, M., Jaafar, H., Aloui, A., \& Souissi, N. (2018). Soccerrelated performance in eumenorrheic Tunisian high-level soccer players: effects of menstrual cycle phase and moment of day. The Journal of Sports Medicine and Physical Fitness, 58(4), 497-502. https://doi.org/10.23736/S0022-4707.17.06958-4

Vescovi, J. D. (2011). The menstrual cycle and anterior cruciate ligament injury risk: Implications of menstrual cycle variability. Sports Medicine, 41(2), 91-101. https://doi.org/10.2165/11538570-000000000-00000

Warren, A. J., O'Brien, M. S., \& Smith, D. B. (2018). Reliability of three urinalysis methods used in the assessment of hydration. International Journal of Sport, Exercise and Health Research, 2(2), 100-105.

Zubac, D., Sekulić, D., \& Karninčić, H. (2014). Reagent strips are reliable and valid measure in defining status of (de)hydration among male junior boxers. Research in Physical Education, Sport and Health, 3(2), 25-28. 